ORIGINAL ARTICLE

\section{Liraglutide and Renal Outcomes in Type 2 Diabetes}

\author{
Johannes F.E. Mann, M.D., David D. Ørsted, M.D., Ph.D., \\ Kirstine Brown-Frandsen, M.D., Steven P. Marso, M.D., \\ Neil R. Poulter, F.Med.Sci., Søren Rasmussen, Ph.D., Karen Tornøe, M.D., Ph.D., \\ Bernard Zinman, M.D., and John B. Buse, M.D., Ph.D., \\ for the LEADER Steering Committee and Investigators*
}

ABSTRACT

\section{BACKGROUND}

In a randomized, controlled trial that compared liraglutide, a glucagon-like peptide 1 analogue, with placebo in patients with type 2 diabetes and high cardiovascular risk who were receiving usual care, we found that liraglutide resulted in lower risks of the primary end point (nonfatal myocardial infarction, nonfatal stroke, or death from cardiovascular causes) and death. However, the long-term effects of liraglutide on renal outcomes in patients with type 2 diabetes are unknown.

\section{METHODS}

We report the prespecified secondary renal outcomes of that randomized, controlled trial in which patients were assigned to receive liraglutide or placebo. The secondary renal outcome was a composite of new-onset persistent macroalbuminuria, persistent doubling of the serum creatinine level, end-stage renal disease, or death due to renal disease. The risk of renal outcomes was determined with the use of time-to-event analyses with an intention-to-treat approach. Changes in the estimated glomerular filtration rate and albuminuria were also analyzed.

\section{RESULTS}

A total of 9340 patients underwent randomization, and the median follow-up of the patients was 3.84 years. The renal outcome occurred in fewer participants in the liraglutide group than in the placebo group (268 of 4668 patients vs. 337 of 4672; hazard ratio, $0.78 ; 95 \%$ confidence interval [CI], 0.67 to $0.92 ; \mathrm{P}=0.003)$. This result was driven primarily by the new onset of persistent macroalbuminuria, which occurred in fewer participants in the liraglutide group than in the placebo group (161 vs. 215 patients; hazard ratio, $0.74 ; 95 \% \mathrm{CI}, 0.60$ to $0.91 ; \mathrm{P}=0.004$ ). The rates of renal adverse events were similar in the liraglutide group and the placebo group (15.1 events and 16.5 events per 1000 patient-years), including the rate of acute kidney injury (7.1 and 6.2 events per 1000 patient-years, respectively).

\section{CONCLUSIONS}

This prespecified secondary analysis shows that, when added to usual care, liraglutide resulted in lower rates of the development and progression of diabetic kidney disease than placebo. (Funded by Novo Nordisk and the National Institutes of Health; LEADER ClinicalTrials.gov number, NCT01179048.)
From KfH Kidney Center, Munich, and Friedrich Alexander University of Erlangen, Erlangen - both in Germany (J.F.E.M.); Novo Nordisk, Bagsvaerd, Denmark (D.D.Ø., K.B.-F., S.R., K.T.); University of Texas Southwestern Medical Center, Dallas (S.P.M.); Imperial College London, London (N.R.P.); Lunenfeld-Tanenbaum Research Institute, Mt. Sinai Hospital, University of Toronto, Toronto (B.Z.); and University of North Carolina School of Medicine, Chapel Hill (J.B.B.). Address reprint requests to Dr. Mann at the KfH Kidney Center, 15 Isoldenstr., Munich 80804 , Germany, or at prof.j.mann@gmail.com.

*A complete list of the steering committee and investigators in the Liraglutide Effect and Action in Diabetes: Evaluation of Cardiovascular Outcome Results (LEADER) trial is provided in the Supplementary Appendix, available at NEJM.org.

N Engl J Med 2017;377:839-48.

DOI: 10.1056/NEJMoal616011

Copyright (๑) 2017 Massachusetts Medical Society. 
P ERSONS WITH TYPE 2 DIABETES MELLITUS, particularly those with evidence of cardiovascular disease, are at high risk for chronic kidney disease. ${ }^{1,2}$ In most countries, diabetic kidney disease is reported to be the leading cause of dialysis-dependent chronic kidney disease. ${ }^{3}$ Efforts to blunt the global increase in the prevalence of end-stage renal disease therefore include the prevention of new-onset diabetic nephropathy and the prevention of progression of established diabetic nephropathy.

Improved glycemic control has been shown to reduce the occurrence of early diabetic nephropathy..$^{4-7}$ In patients with established diabetic nephropathy, blood-pressure control and inhibition of the renin-angiotensin system are pivotal to slowing a progressive reduction in the glomerular filtration rate. ${ }^{8}$

Some glucose-lowering agents appear to have glucose-independent effects on diabetic nephropathy and its progression. Data from studies investigating the dipeptidyl peptidase 4 (DPP-4) inhibitor linagliptin and the human glucagon-like peptide 1 (GLP-1) analogue liraglutide, as well as sodium-glucose cotransporter 2 (SGLT2) inhibitors, suggest that these drugs exert beneficial effects in experimental models of kidney injury. ${ }^{9-11}$ However, in long-term studies, no clinically meaningful benefits on renal outcomes have emerged with these medications as compared with placebo, except for a convincing benefit among patients who have received the SGLT2 inhibitor empagliflozin. ${ }^{11}$

In short-term studies, liraglutide improved glycemic control, reduced urinary albumin excretion, and increased urinary sodium excretion..$^{12,13}$ However, the long-term effects of liraglutide on the development and progression of diabetic kidney disease are unknown. The Liraglutide Effect and Action in Diabetes: Evaluation of Cardiovascular Outcome Results (LEADER) trial recently showed lower risks of cardiovascular outcomes, death from any cause, and microvascular outcomes with liraglutide than with placebo. ${ }^{14}$ The lower risk of microvascular outcomes was driven by renal outcomes. Therefore, we report here the results of a prespecified analysis of the effects of liraglutide on renal outcomes.
METHODS

\section{TRIAL DESIGN AND OVERSIGHT}

The trial design and methods have been published previously. ${ }^{15}$ The trial protocol, available with the full text of this article at NEJM.org, was approved by the institutional review board or ethics committee at each participating center, and all the patients provided written informed consent. In brief, in this double-blind, placebocontrolled trial, patients with type 2 diabetes and a high risk of cardiovascular disease were randomly assigned, in a 1:1 ratio, to receive liraglutide or matching placebo, in addition to usual care. The disposition of the trial participants has been published previously. ${ }^{15}$ Given the regulatory request to provide data on the use of liraglutide in patients with stage 4 chronic kidney disease (estimated glomerular filtration rate [GFR], 15 to $<30 \mathrm{ml}$ per minute per $1.73 \mathrm{~m}^{2}$ of body-surface area), approximately 220 such patients were included in the trial. The minimum planned follow-up of the patients receiving the trial regimen was 42 months, and the maximum planned followup was 60 months, with an additional 30 days of follow-up after the trial regimen was completed.

All the authors had access to the final trial results and vouch for the fidelity of the trial to the protocol. The first two authors drafted the manuscript of this article, which was revised and approved by all the authors, who also vouch for the accuracy and completeness of the data and analyses reported.

\section{MICROVASCULAR AND RENAL OUTCOMES}

The prespecified secondary microvascular outcome was a composite of renal and retinal outcomes. ${ }^{15}$ In this article, we focus on the renal outcomes, which were more common than the retinal events (Table S1 in the Supplementary Appendix, available at NEJM.org). The composite renal outcome consisted of new-onset persistent macroalbuminuria, persistent doubling of the serum creatinine level and an estimated GFR of $45 \mathrm{ml}$ or less per minute per $1.73 \mathrm{~m}^{2}$ of bodysurface area (hereafter referred to as persistent doubling of the serum creatinine level), the need for continuous renal-replacement therapy (end- 
stage renal disease) with no reversible cause of the renal disease, or death from renal disease. Macroalbuminuria was defined as a concentration of more than $300 \mathrm{mg}$ of albumin in a 24-hour urine sample or as a concentration of more than $300 \mathrm{mg}$ of albumin per gram of creatinine in a first morning sample. To confirm the presence of persistent macroalbuminuria or doubling of the serum creatinine level, a second measurement was mandatory.

Renal outcomes were ascertained every 12 months and at the end of the trial. All the abovementioned renal outcomes were adjudicated by an independent committee of experts who were unaware of the trial-group assignments, as described previously. ${ }^{14,15}$ Additional nonadjudicated renal outcomes and adverse-event reporting are described in the Supplementary Methods section in the Supplementary Appendix.

\section{LABORATORY ASSESSMENTS}

Laboratory tests were performed centrally (at ICON in Warrington, PA). Creatinine levels (both urinary and serum) were measured enzymatically, calibrated to isotope dilution mass spectrometry values (Roche-Hitachi), and the urinary albumin concentration was assessed by means of immunoprecipitation (Roche-Hitachi). The serum creatinine level and the albumin-to-creatinine ratio were measured and calculated at randomization, annually, and at trial completion; the serum creatinine level was also measured at month 6 . The GFR was estimated with the four-component Modification of Diet in Renal Disease formula, as specified in the trial protocol ${ }^{16}$ Microalbuminuria (or a high urinary albumin level) was defined as a concentration of 30 to $300 \mathrm{mg}$ of albumin per gram of creatinine, and macroalbuminuria (or a very high urinary albumin level) as a concentration of more than $300 \mathrm{mg}$ of albumin per gram of creatinine. If the urinary albumin measurements were below the lower limit of quantification ( $5 \mathrm{mg}$ per liter), a value of $2.5 \mathrm{mg}$ per liter was imputed for statistical analysis.

\section{STATISTICAL ANALYSIS}

The statistical analysis plan is available with the protocol, ${ }^{14}$ and additional details are provided in the Supplementary Methods section in the Supplementary Appendix. In this secondary analysis, the required sample size was based on the primary cardiovascular outcome. ${ }^{15}$ Although the trial was not powered for renal outcomes, we calculated post hoc that the trial had $85 \%$ power to detect a risk of the composite renal outcome that was $22 \%$ lower in the liraglutide group than in the placebo group. Renal outcomes were not included in the hierarchical testing strategy that was used for the primary cardiovascular outcome. All the analyses were performed at the nominal alpha level of 0.05 without correction for multiple hypothesis testing.

In prespecified time-to-event analyses we applied a Cox proportional-hazards model with trial group as a covariate. Sensitivity analyses were performed with death as a competing risk. All the patients who underwent randomization were included and followed from the time of randomization until death or the end of followup, whichever came first.

Prespecified between-group comparisons were performed at 36 months, which was the last trial visit at which laboratory testing was performed at the same time point in the entire population. We estimated trial-group ratios for the estimated GFR and the urinary albumin-tocreatinine ratio using a mixed-effects model for repeated measures on log-transformed values (see the Supplementary Methods section in the Supplementary Appendix). Subgroups of patients who were at increased renal risk (according to the estimated GFR and urinary albumin-to-creatinine ratio at baseline) were defined before unblinding.

\section{RESULTS}

\section{TRIAL POPULATION}

A total of 9340 patients underwent randomization, with 4668 patients being assigned to receive liraglutide and 4672 to receive placebo. The median follow-up of the patients was 3.84 years, and the mean percentage of time that patients were taking the trial regimen was $83.0 \%$.

The characteristics of the patients at baseline were generally balanced between the trial groups (Tables S2 and S3 in the Supplementary Appen- 


\begin{tabular}{|c|c|c|c|c|c|}
\hline Outcome & $\begin{array}{l}\text { Liraglutide } \\
(\mathrm{N}=4668)\end{array}$ & $\begin{array}{l}\text { Placebo } \\
(\mathrm{N}=4672)\end{array}$ & $\begin{array}{c}\text { Total } \\
(\mathrm{N}=9340)\end{array}$ & $\begin{array}{l}\text { Hazard Ratio } \\
(95 \% \mathrm{Cl})\end{array}$ & P Value \\
\hline \multicolumn{6}{|c|}{ no. of patients (rate per 1000 patient-yr of observation) } \\
\hline Composite renal outcome & $268(15.0)$ & $337(19.0)$ & $605(17.0)$ & $0.78(0.67-0.92)$ & 0.003 \\
\hline \multicolumn{6}{|l|}{ Components of composite renal outcome $\dagger^{\dagger}$} \\
\hline New-onset persistent macroalbuminuria & $161(9.0)$ & $215(12.1)$ & $376(10.6)$ & $0.74(0.60-0.91)$ & 0.004 \\
\hline $\begin{array}{l}\text { Persistent doubling of serum creatinine } \\
\text { level }\end{array}$ & $87(4.9)$ & $97(5.5)$ & $184(5.2)$ & $0.89(0.67-1.19)$ & 0.43 \\
\hline Renal-replacement therapy & $56(3.1)$ & $64(3.6)$ & $120(3.4)$ & $0.87(0.61-1.24)$ & 0.44 \\
\hline Death due to renal disease & $8(0.4)$ & $5(0.3)$ & $13(0.4)$ & $1.59(0.52-4.87)$ & 0.41 \\
\hline
\end{tabular}

* There were 17,822 patient-years of observation in the liraglutide group and 17,741 in the placebo group. All the events were adjudicated. Hazard ratios and $\mathrm{P}$ values were estimated with the use of a Cox proportional-hazards model with trial group as a covariate.

$\uparrow$ The composite renal outcome consisted of new-onset persistent macroalbuminuria, persistent doubling of the serum creatinine level and an estimated glomerular filtration rate of $45 \mathrm{ml}$ or less per minute per $1.73 \mathrm{~m}^{2}$ of body-surface area (referred to as persistent doubling of the serum creatinine level), the need for continuous renal-replacement therapy (end-stage renal disease), or death due to renal disease. One patient who had macroalbuminuria at baseline had an event of new-onset persistent macroalbuminuria that was confirmed by adjudication after the patient had regression to microalbuminuria earlier in the trial.

dix). The mean age of the patients was 64 years, and the mean blood pressure $136 / 77 \mathrm{~mm} \mathrm{Hg}$. The mean estimated GFR was $80 \mathrm{ml}$ per minute per $1.73 \mathrm{~m}^{2}$; a total of $20.7 \%$ of the patients had an estimated GFR of 30 to $59 \mathrm{ml}$ per minute per $1.73 \mathrm{~m}^{2}$, and $2.4 \%$ had an estimated GFR of less than $30 \mathrm{ml}$ per minute per $1.73 \mathrm{~m}^{2}$. Microalbuminuria and macroalbuminuria, as assessed at randomization, were present in $26.3 \%$ and $10.5 \%$ of the patients, respectively. A total of 8632 patients received antihypertensive agents, and 7741 received renin-angiotensin system blockers.

\section{COMPOSITE RENAL OUTCOME}

The prespecified composite renal outcome occurred in fewer patients in the liraglutide group than in the placebo group (268 patients [5.7\%] vs. 337 [7.2\%]; hazard ratio, 0.78; 95\% confidence interval [CI], 0.67 to 0.92; $\mathrm{P}=0.003$ ) (Table 1 and Fig. 1A). New-onset persistent macroalbuminuria occurred in fewer patients in the liraglutide group than in the placebo group (161 patients [3.4\%] vs. 215 [4.6\%]; hazard ratio, 0.74; 95\% CI, 0.60 to $0.91 ; \mathrm{P}=0.004$ ) (Fig. $1 \mathrm{~B}$ ). The risks of persistent doubling of the serum creatinine level and of end-stage renal disease (use of renalreplacement therapy) did not differ significantly between groups (Table 1 and Fig. 1C and 1D). There were few cases of death due to renal disease ( 8 patients in the liraglutide group and 5 in the placebo group).
Sensitivity analyses, in which death from any cause was considered to be a competing risk, yielded similar results (Table S4 in the Supplementary Appendix). Similarly, analyses that took into account the differences between groups in the use of inhibitors of the renin-angiotensin system, the glycated hemoglobin level, body weight, and blood pressure did not materially alter the results (Tables S5 through S8 in the Supplementary Appendix).

\section{COMPOSITE RENAL OUTCOME IN SUBGROUPS}

The prespecified subgroups of patients with an elevated renal risk at baseline included 3422 patients with microalbuminuria or macroalbuminuria, 2158 with an estimated GFR of less than $60 \mathrm{ml}$ per minute per $1.73 \mathrm{~m}^{2}$, and 1130 with both an estimated GFR of less than $60 \mathrm{ml}$ per minute per $1.73 \mathrm{~m}^{2}$ and microalbuminuria or macroalbuminuria (Fig. 2, and Table S9 in the Supplementary Appendix). The effect of liraglutide on the composite renal outcome appeared to be independent of subgroups (Fig. 2). In the subgroup of patients with microalbuminuria or macroalbuminuria, the composite renal outcome occurred in fewer patients in the liraglutide group than in the placebo group (230 of 1684 patients [13.7\%] vs. 283 of 1738 [16.3\%]; hazard ratio, $0.81 ; 95 \% \mathrm{CI}, 0.68$ to $0.96 ; \mathrm{P}=0.02$ ). In the subgroup of patients with an estimated GFR of less than $60 \mathrm{ml}$ per minute per $1.73 \mathrm{~m}^{2}$, the 


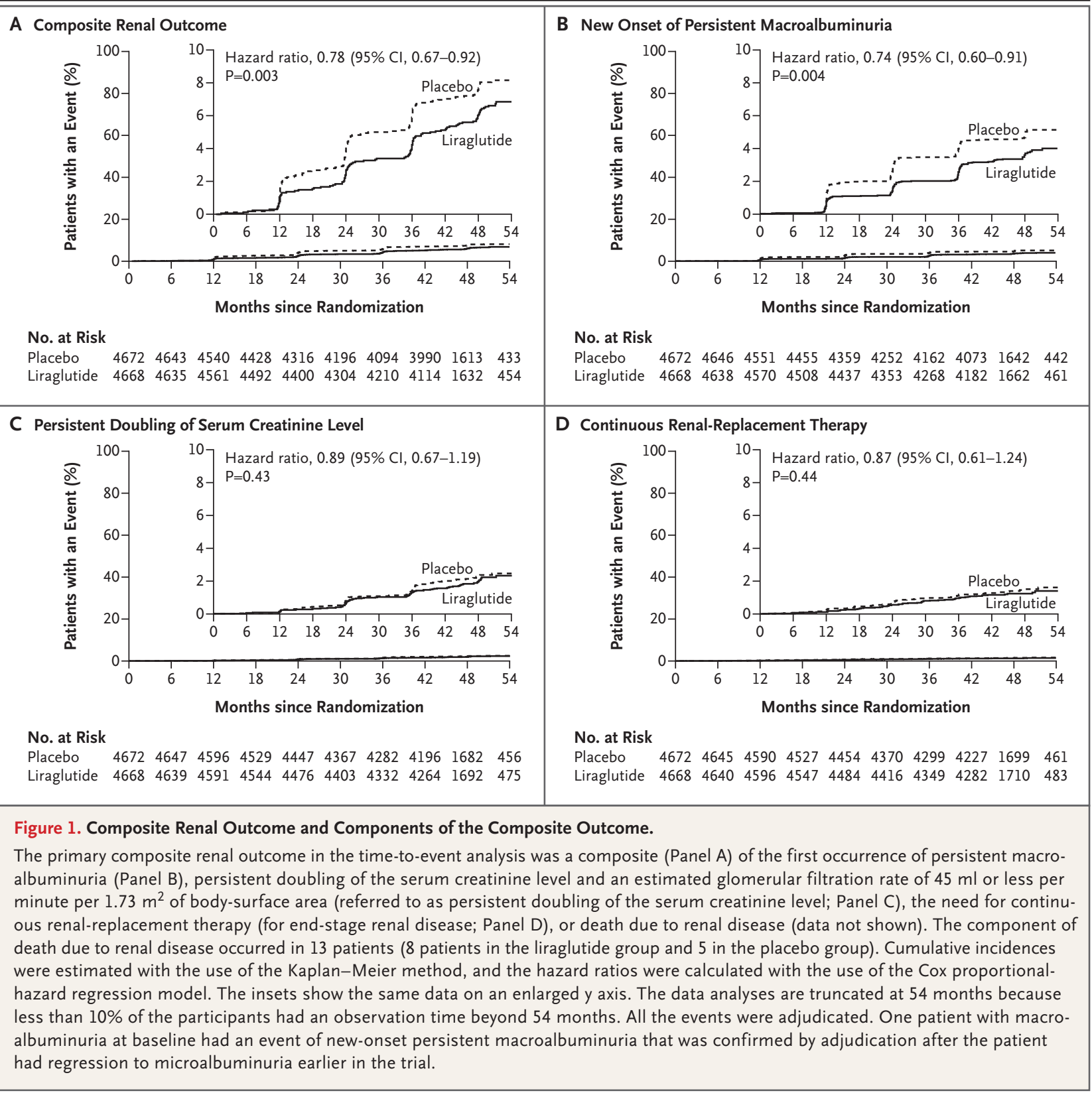

composite renal outcome occurred in 146 of 1116 patients (13.1\%) in the liraglutide group and in 156 of $1042(15.0 \%)$ in the placebo group (hazard ratio, $0.84 ; 95 \% \mathrm{CI}, 0.67$ to $1.05 ; \mathrm{P}=0.13$ ). In patients with both an estimated GFR of less than $60 \mathrm{ml}$ per minute per $1.73 \mathrm{~m}^{2}$ and microalbuminuria or macroalbuminuria, the composite renal outcome occurred in 131 of 583 patients $(22.5 \%)$ in the liraglutide group and in 141 of $547(25.8 \%)$ in the placebo group (hazard ratio, $0.81 ; 95 \% \mathrm{CI}, 0.64$ to $1.03 ; \mathrm{P}=0.09$ ).

\section{RENAL FUNCTION OVER TIME}

The estimated GFR declined continuously (Fig. 3A), but the decline was slightly slower in the liraglutide group than in the placebo group (estimated trial-group ratio at 36 months, 1.02; 95\% CI, 1.00 to $1.03 ; \mathrm{P}=0.01$, corresponding to a $2 \%$ less decrease with liraglutide). The decrease in the estimated GFR at 36 months was $7.44 \mathrm{ml}$ per minute per $1.73 \mathrm{~m}^{2}$ in the liraglutide group, as compared with $7.82 \mathrm{ml}$ per minute per $1.73 \mathrm{~m}^{2}$ in the placebo group. The urinary albumin-to- 


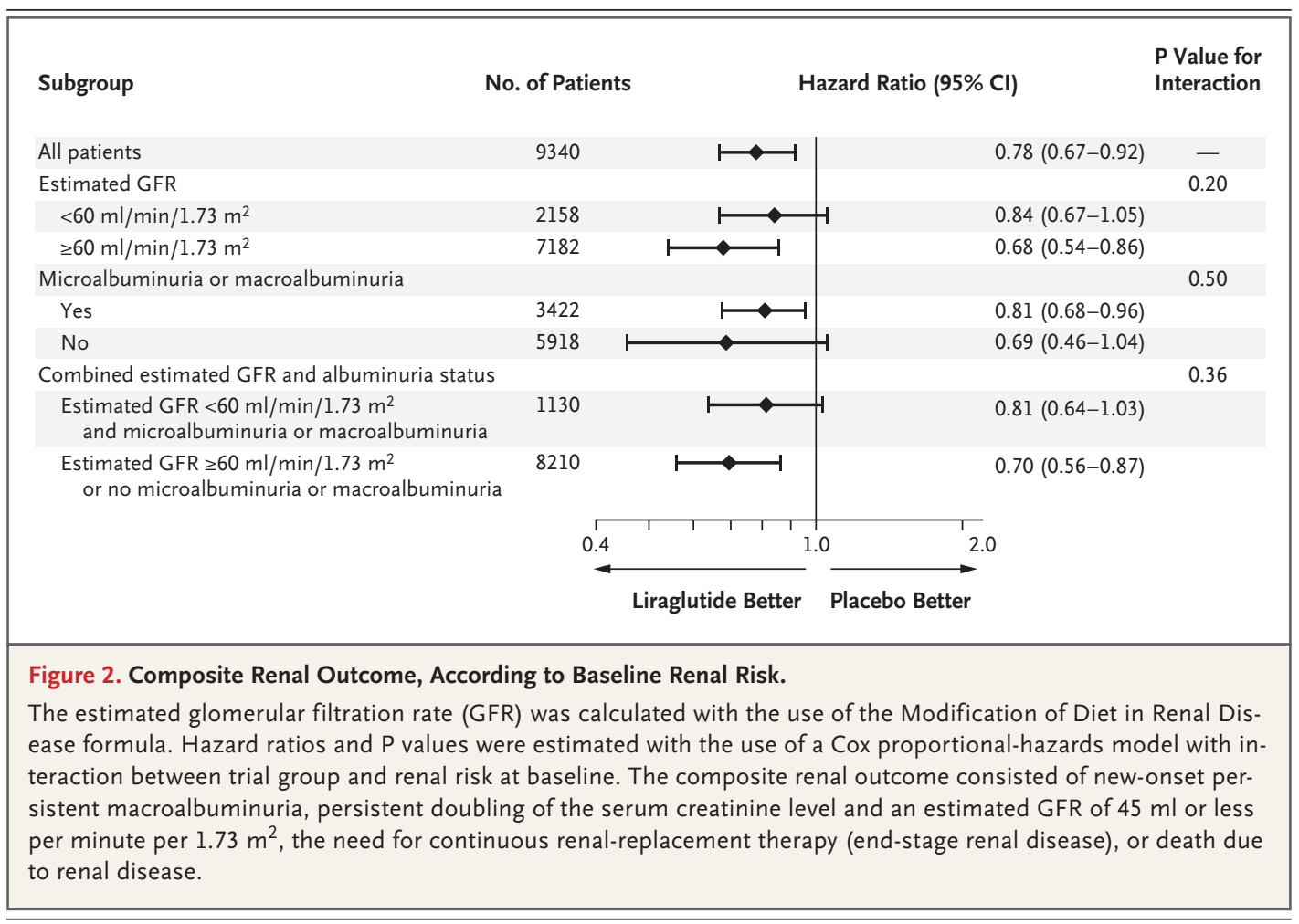

creatinine ratio increased less in the liraglutide group, yielding a 17\% lower urinary albumin-tocreatinine ratio at 36 months in favor of liraglutide (estimated trial-group ratio, $0.83 ; 95 \% \mathrm{CI}$, 0.79 to 0.88 ; $\mathrm{P}<0.001$ ) (Fig. $3 \mathrm{~B}$ ). The estimated increase in the urinary albumin-to-creatinine ratio at 36 months was $1.8 \mathrm{mg}$ of albumin per gram of creatinine in the liraglutide group, as compared with $6.3 \mathrm{mg}$ of albumin per gram of creatinine in the placebo group (Fig. 3B); in an analysis that excluded values outside the quantification range, the results were similar (Table S10A and S10B in the Supplementary Appendix). The smaller increase in albuminuria at 36 months in the liraglutide group than in the placebo group was independent of the baseline estimated GFR and baseline albuminuria (Table S10A and S10B and Fig. S1A and S1B in the Supplementary Appendix).

When results were stratified according to the estimated GFR at baseline, the decrease in the estimated GFR in patients with a baseline estimated GFR of 30 to $59 \mathrm{ml}$ per minute per $1.73 \mathrm{~m}^{2}$ was $2 \mathrm{ml}$ per minute per $1.73 \mathrm{~m}^{2}$ in the liraglutide group, as compared with $4 \mathrm{ml}$ per minute per $1.73 \mathrm{~m}^{2}$ in the placebo group (estimated trialgroup ratio in favor of liraglutide, 1.07; 95\% CI,
1.04 to 1.10 ; P <0.001) (Fig. 4, and Fig. S2 in the Supplementary Appendix). Results did not differ significantly between groups in patients with a baseline estimated GFR of $60 \mathrm{ml}$ or more per minute per $1.73 \mathrm{~m}^{2}$ or with an estimated GFR of less than $30 \mathrm{ml}$ per minute per $1.73 \mathrm{~m}^{2}$ (there were few patients in the latter group). When analyzed according to albuminuria at baseline, the decrease in the estimated GFR was smaller in the liraglutide group than in the placebo group in patients with macroalbuminuria $(\mathrm{P}=0.01)$ but did not differ significantly in those with microalbuminuria $(\mathrm{P}=0.24)$ or normoalbuminuria $(P=0.60)$ (data not shown).

\section{OTHER OUTCOMES AND ADVERSE EVENTS}

Outcomes that were not prespecified included the composite of the doubling of the serum creatinine level or the use of renal-replacement therapy; there were no significant differences between the randomized groups (Table S11 in the Supplementary Appendix). New-onset microalbuminuria occurred in fewer patients in the liraglutide group than in the placebo group (2293 patients [49.1\%] vs. 2498 [53.5\%]; hazard ratio, 0.87; 95\% CI, 0.83 to 0.93 ; $\mathrm{P}<0.001$ ).

The rates of renal adverse events were similar 
in the liraglutide group and the placebo group (15.1 events and 16.5 events per 1000 patientyears), including the rate of acute kidney injury (7.1 events and 6.2 events per 1000 patient-years, respectively), according to adverse-event reporting. The similarity in the rates of adverse events also applies to subgroups defined according to the estimated GFR at baseline. Details are provided in Table S12A and S12B in the Supplementary Appendix.

\section{DISCUSSION}

Among patients who were receiving usual care, liraglutide resulted in significantly lower rates of renal outcomes than placebo among patients with type 2 diabetes who were at high cardiovascular risk. This result was driven mainly by a lower incidence of macroalbuminuria in the liraglutide group than in the placebo group. There were nonsignificantly lower risks of the doubling of the serum creatinine level and of end-stage renal disease with liraglutide than with placebo during up to 5 years of follow-up.

New-onset persistent macroalbuminuria is an effect that is typically associated with subsequent progressive reductions in the GFR in patients with type 2 diabetes. ${ }^{17}$ For example, in ONTARGET (Ongoing Telmisartan Alone and in Combination with Ramipril Global Endpoint Trial) and TRANSCEND (Telmisartan Randomized Assessment Study in ACE Intolerant Subjects with Cardiovascular Disease), new-onset macroalbuminuria was associated with a risk of end-stage renal disease or doubling of the serum creatinine level that was 3 to 5 times as high as the risk among patients in whom new-onset macroalbuminuria did not develop. ${ }^{18}$ Similar renal outcomes associated with new-onset macroalbuminuria were reported in the ADVANCE (Action in Diabetes and Vascular Disease: Preterax and Diamicron Modified Release Controlled Evaluation) trial $^{19}$ and supported by meta-regression analyses. ${ }^{17}$ Macroalbuminuria is also a risk factor for cardiovascular events. . $^{17,20}$

In this present trial, the risks of doubling of the serum creatinine level and end-stage renal disease did not differ significantly between the liraglutide group and the placebo group, possibly owing to the moderate decline in the estimated GFR observed in this cohort and the few patients who had advanced kidney disease at

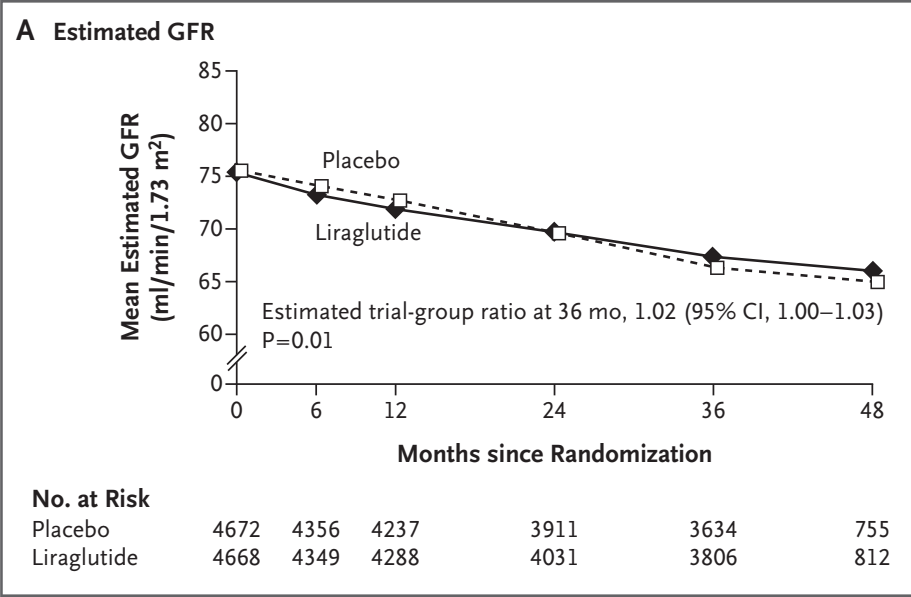

B Urinary Albumin-to-Creatinine Ratio

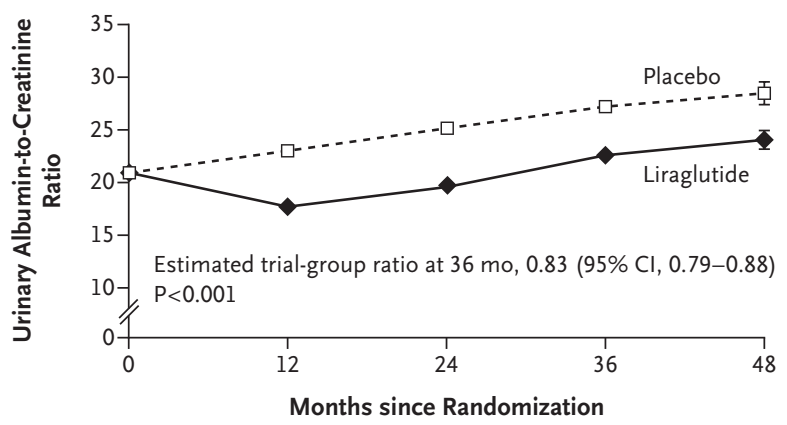

No. at Risk

Placebo

Liraglutide

\section{9}

4578

4103
4167

3789
3934

3509

3686

730

786

Figure 3. Changes in the Estimated GFR and Urinary Albumin-to-Creatinine Ratio.

Panel A shows the estimated GFR, and Panel B the urinary albumin-tocreatinine ratio (with albumin measured in milligrams and creatinine measured in grams). Geometric means were estimated for the urinary albuminto-creatinine ratio with the use of a linear mixed model for log-transformed assessment, with accounting for repeated measures. Trial-group ratios were estimated with the use of a mixed-effect model for repeated measures on log-transformed values. Interaction between visit and, respectively, trial group, sex, geographic region, and use of antidiabetic therapy at baseline were included as fixed effects, and interaction between visit and baseline log-estimated GFR or baseline urinary albumin-to-creatinine ratio and age at baseline were included as covariates. The values for the urinary albuminto-creatinine ratio that were outside the range of quantification were imputed (see the Supplementary Methods section in the Supplementary Appendix).

randomization. The initial changes in the estimated GFR are difficult to interpret, ${ }^{21}$ but there may be a potential slowing in the reduction in the estimated GFR with liraglutide in patients with a low baseline estimated GFR.

The mechanism behind the effect of liraglutide on the renal outcomes is unclear. Statistical adjustment for the glycated hemoglobin level at 


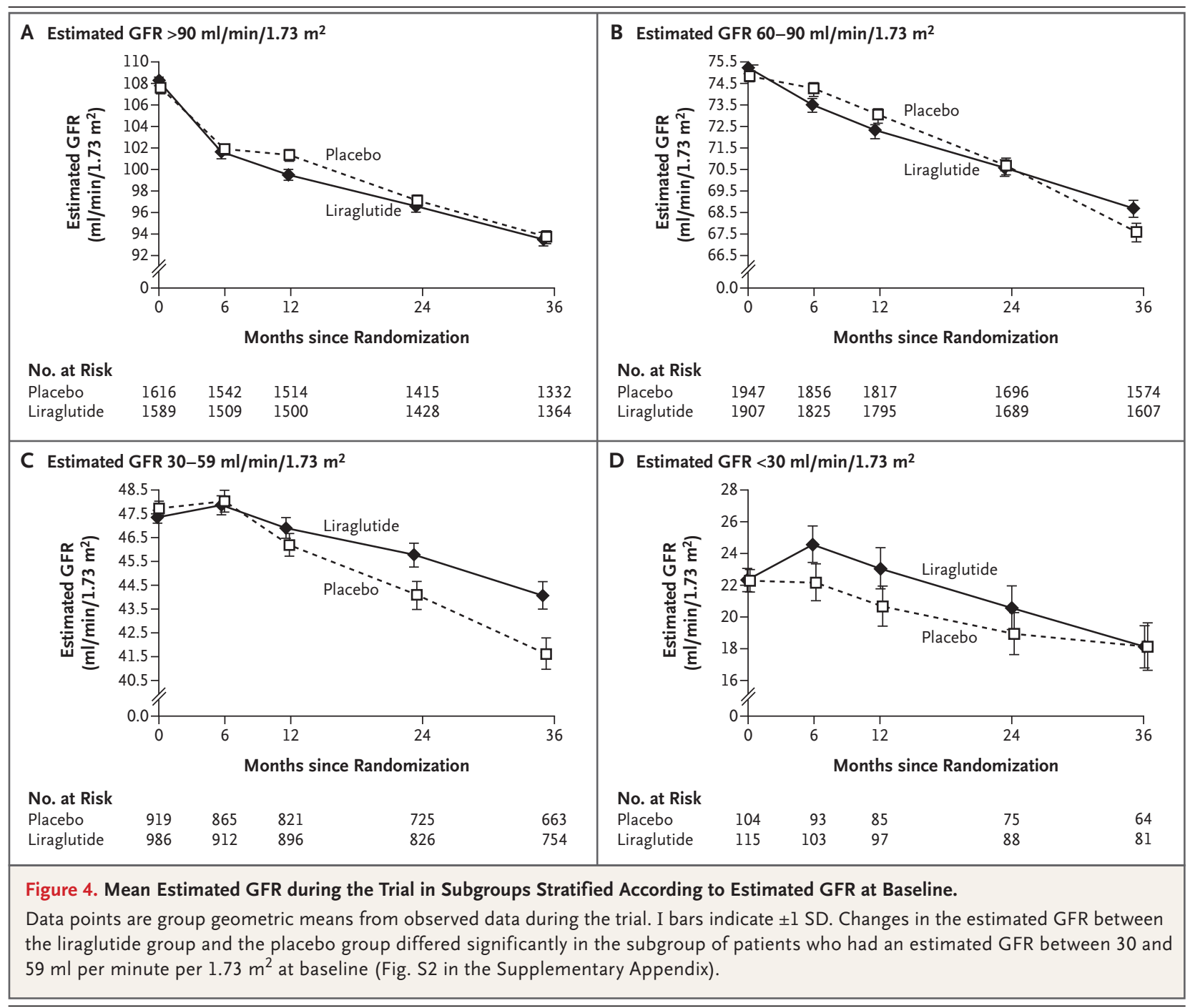

baseline and during the trial did not materially alter the effect of liraglutide on renal outcomes. However, in a meta-analysis, intensified glucose control was associated with a lower incidence of new macroalbuminuria than was usual care. ${ }^{4}$ In the United Kingdom Prospective Diabetes Study (UKPDS), a 1-percentage-point difference in the glycated hemoglobin level between groups was associated with a consistent benefit on microvascular outcomes only, after 10 years of followup. ${ }^{22}$ In the ACCORD (Action to Control Cardiovascular Risk in Diabetes) trial, a difference of 0.8 percentage points in the glycated hemoglobin level was associated with a much earlier benefit on macroalbuminuria but no change in the risks of doubling of the serum creatinine level or end-stage renal disease with 4 years of follow-up. ${ }^{23}$ The ADVANCE trial showed a difference of 0.8 percentage points in the glycated hemoglobin level and a substantial benefit with regard to new-onset albuminuria over a 5-year follow-up with no significant change in the risk of a doubling of the serum creatinine level but a tendency toward a lower risk of end-stage renal disease. ${ }^{24}$ Other large, randomized trials involving patients with type 2 diabetes have also reported microvascular events..$^{25-28}$ As in the present trial, these trials have shown between-group differences in the glycated hemoglobin level of between 0.2 and 0.4 percentage points, and benefits of active compounds on albuminuria, but not on other renal outcomes. In contrast, the EMPA-REG OUTCOME trial showed that empagliflozin resulted in significantly lower risks 
than placebo of progression to macroalbuminuria, the doubling of the serum creatinine level, and despite small numbers, end-stage renal disease. ${ }^{11,29}$

It appears to be unlikely that the moderate between-group differences in systolic blood pressure and body weight can fully explain the effect on renal outcomes. In an analysis that took into account those differences, the composite renal outcome still occurred less frequently with liraglutide than with placebo. The mechanisms behind the renal effects of liraglutide are probably multifactorial. Most studies investigating the effect of GLP-1 treatment on renal hemodynamic variables in patients with type 2 diabetes have shown neutral effects. ${ }^{12,13}$ Preclinical studies have shown convincingly that GLP-1 therapy decreases the level of inflammation and oxidative stress and prevents diabetic nephropathy and acute kidney injury. ${ }^{10,30}$ Furthermore, a recent randomized trial involving patients with type 2 diabetes and albuminuria showed that liraglutide treatment reduced levels of inflammatory biomarkers. ${ }^{31}$ Hence, it is possible that preservation of renal function and the antialbuminuric effects of liraglutide may be due to antiinflammatory effects rather than renal hemodynamic effects.

There are some limitations to the present analysis. Although the trial prespecified renal outcomes as secondary outcomes, it was not powered for analyses of the individual renal outcomes. Also, patients were followed for 3.5 to 5.0 years, and thus we cannot extrapolate to longer-term effects. The trial design prevents us from ascertaining the extent to which the active drug, glucose control, or other factors (including concomitant medications) explain outcomes. A significantly lower risk of renal outcomes was still observed when the analysis was adjusted for change in the glycated hemoglobin level, body weight, and blood pressure. That said, in the absence of head-to-head comparisons, other direct or indirect drug effects cannot be ruled out. Nonetheless, concomitant medications were more frequently added in the placebo group during the trial than in the liraglutide group. ${ }^{14}$

In conclusion, in this secondary analysis, among patients with type 2 diabetes at high risk for cardiovascular disease who were receiving usual care, liraglutide resulted in a lower risk of the composite renal outcome than placebo, primarily owing to a lower rate of new-onset persistent macroalbuminuria.

Supported by Novo Nordisk and by a grant (UL1TR001111, to Dr. Buse) from the National Institutes of Health.

Disclosure forms provided by the authors are available with the full text of the article at NEJM.org.

We thank Drs. Florian Baeres and Thomas Idorn (Novo Nordisk) for scientific input, and the participants and trial-site staff.

\section{REFERENCES}

1. Ritz E, Orth SR. Nephropathy in patients with type 2 diabetes mellitus. N Engl J Med 1999;341:1127-33.

2. Alegre-Díaz J, Herrington W, López Cervantes $M$, et al. Diabetes and causespecific mortality in Mexico City. N Engl J Med 2016;375:1961-71.

3. Molitch ME, Adler AI, Flyvbjerg A, et al. Diabetic kidney disease: a clinical update from Kidney Disease: Improving Global Outcomes. Kidney Int 2015;87:20-30.

4. Coca SG, Ismail-Beigi $\mathrm{F}$, Haq $\mathrm{N}$, Krumholz HM, Parikh CR. Role of intensive glucose control in development of renal end points in type 2 diabetes mellitus: systematic review and meta-analysis. Arch Intern Med 2012;172:761-9.

5. Perkovic V, Heerspink HL, Chalmers $\mathrm{J}$, et al. Intensive glucose control improves kidney outcomes in patients with type 2 diabetes. Kidney Int 2013;83:517-23.

6. UK Prospective Diabetes Study (UKPDS) Group. Effect of intensive bloodglucose control with metformin on complications in overweight patients with type 2 diabetes (UKPDS 34). Lancet 1998; 352:854-65.

7. UK Prospective Diabetes Study
(UKPDS) Group. Intensive blood-glucose control with sulphonylureas or insulin compared with conventional treatment and risk of complications in patients with type 2 diabetes (UKPDS 33). Lancet 1998;352:837-53.

8. Stevens PE, Levin A. Evaluation and management of chronic kidney disease: synopsis of the Kidney Disease: Improving Global Outcomes 2012 clinical practice guideline. Ann Intern Med 2013;158: 825-30.

9. Zeisberg M, Zeisberg EM. Evidence for antifibrotic incretin-independent effects of the DPP-4 inhibitor linagliptin. Kidney Int 2015;88:429-31.

10. Fujita H, Morii T, Fujishima $\mathrm{H}$, et al. The protective roles of GLP-1R signaling in diabetic nephropathy: possible mechanism and therapeutic potential. Kidney Int 2014;85:579-89.

11. Wanner C, Inzucchi SE, Lachin JM, et al. Empagliflozin and progression of kidney disease in type 2 diabetes. $\mathrm{N}$ Engl J Med 2016;375:323-34.

12. von Scholten BJ, Lajer M, Goetze JP, Persson F, Rossing P. Time course and mechanisms of the anti-hypertensive and renal effects of liraglutide treatment. Diabet Med 2015;32:343-52.

13. Skov J, Pedersen M, Holst JJ, et al. Short-term effects of liraglutide on kidney function and vasoactive hormones in type 2 diabetes: a randomized clinical trial. Diabetes Obes Metab 2016;18:581-9.

14. Marso SP, Daniels GH, Brown-Frandsen $\mathrm{K}$, et al. Liraglutide and cardiovascular outcomes in type 2 diabetes. $\mathrm{N}$ Engl J Med 2016;375:311-22.

15. Marso SP, Poulter NR, Nissen SE, et al. Design of the Liraglutide Effect and Action in Diabetes: Evaluation of Cardiovascular Outcome Results (LEADER) trial. Am Heart J 2013;166(5):823-30.e5.

16. Stevens LA, Coresh J, Greene T, Levey AS. Assessing kidney function - measured and estimated glomerular filtration rate. N Engl J Med 2006;354:2473-83.

17. Fox CS, Matsushita K, Woodward M, et al. Associations of kidney disease measures with mortality and end-stage renal disease in individuals with and without diabetes: a meta-analysis. Lancet 2012; 380:1662-73.

18. Schmieder RE, Mann JF, Schumacher $\mathrm{H}$, et al. Changes in albuminuria predict 
mortality and morbidity in patients with vascular disease. J Am Soc Nephrol 2011; 22:1353-64.

19. Ninomiya T, Perkovic V, de Galan BE, et al. Albuminuria and kidney function independently predict cardiovascular and renal outcomes in diabetes. J Am Soc Nephrol 2009;20:1813-21.

20. Gerstein HC, Mann JF, Yi Q, et al. Albuminuria and risk of cardiovascular events, death, and heart failure in diabetic and nondiabetic individuals. JAMA 2001; 286:421-6.

21. Clase CM, Barzilay J, Gao P, et al. Acute change in glomerular filtration rate with inhibition of the renin-angiotensin system does not predict subsequent renal and cardiovascular outcomes. Kidney Int 2017;91:683-90.

22. Holman RR, Paul SK, Bethel MA, Matthews DR, Neil HAW. 10-Year followup of intensive glucose control in type 2 diabetes. N Engl J Med 2008;359:1577-89.
23. The Action to Control Cardiovascular Risk in Diabetes Study Group. Effects of intensive glucose lowering in type $2 \mathrm{dia}$ betes. N Engl J Med 2008;358:2545-59. 24. Zoungas S, Woodward M, Li Q, et al. Impact of age, age at diagnosis and duration of diabetes on the risk of macrovascular and microvascular complications and death in type 2 diabetes. Diabetologia 2014;57:2465-74.

25. Green JB, Bethel MA, Armstrong PW, et al. Effect of sitagliptin on cardiovascular outcomes in type 2 diabetes. $\mathrm{N}$ Engl Med 2015;373:232-42.

26. Scirica BM, Bhatt DL, Braunwald E, et al. Saxagliptin and cardiovascular outcomes in patients with type 2 diabetes mellitus. N Engl J Med 2013;369:1317-26. 27. White WB, Cannon CP, Heller SR, et al. Alogliptin after acute coronary syndrome in patients with type 2 diabetes. $\mathrm{N}$ Engl Med 2013;369:1327-35.

28. Pfeffer MA, Claggett B, Diaz R, et al.
Lixisenatide in patients with type 2 diabetes and acute coronary syndrome. $\mathrm{N}$ Engl J Med 2015;373:2247-57.

29. Zinman B, Wanner C, Lachin JM, et al. Empagliflozin, cardiovascular outcomes, and mortality in type 2 diabetes. $\mathrm{N}$ Engl J Med 2015;373:2117-28.

30. Kodera R, Shikata K, Kataoka HU, et al. Glucagon-like peptide-1 receptor agonist ameliorates renal injury through its antiinflammatory action without lowering blood glucose level in a rat model of type 1 diabetes. Diabetologia 2011;54:965-78. 31. von Scholten BJ, Persson F, Rosenlund $S$, et al. Effects of liraglutide on cardiovascular risk biomarkers in patients with type 2 diabetes and albuminuria: a subanalysis of a randomized, placebo-controlled, double-blind, crossover trial. Diabetes Obes Metab 2017;19:901-5.

Copyright (C) 2017 Massachusetts Medical Society. 